\title{
Latitudinal variability in the Quaternary development of the Eurasian Ice Sheets - evidence from the marine domain
}

\author{
Berit Oline Hjelstuen and Hans Petter Sejrup \\ Department of Earth Science, University of Bergen, Allegt 41, N-5007 Bergen, Norway
}

\section{Supplemental Material}

\section{RESULTS OF DATA COMPILATION}

The compiled Quaternary isopach map (Fig. 1) is based on merging of previously published isopach maps from the NE Atlantic margin, from Ireland to Svalbard (Faleide et al., 1996; Stoker et al., 2005; Dowdeswell et al., 2010; Ottesen et al., 2012), the North Sea (Ottesen et al., 2018) and the Yermak Plateau (Geissler et al., 2011) (Table S1; Fig. S1). Except for in the region at the mouth of the Kveithola Trough (Lasabuda et al., 2018) no Quaternary isopach map exist in the Nansen Basin, Arctic Ocean. Mapping of Quaternary sediments in the Nansen Basin is, therefore, based on extracting sediment thickness information from published $2 \mathrm{D}$ seismic profiles (Geissler and Jokat, 2004; Jokat and Micksch, 2004; Nikishin et al., 2018) (Fig. S1; Fig. S2, Table S1). Likewise, the thickness map in the Norway Basin (Fig. 1, Fig. S1) is generated from published seismic profiles (Hjelstuen and Andreassen, 2015) and new interpretation of 2D seismic profiles that have been accessible through the DISKOS database (www.diskos.no). Available information from ODP/DSDP sites and shallow borings (Fig. S1) have also been used in order to justify and identify Quaternary sediment thicknesses. Depth conversion, from depth in time to depth in meters, have been performed by using sediment velocities presented in the published papers, or by using information from nearby regions with similar geological setting.

The chronostratigraphy of the pre-Last Glacial Maximum Quaternary sediment package in the region is to a large degree based on paleomagnetic studies, micropaleontology, ${ }^{86 / 87} \mathrm{Sr}$ and to some degree amino acid geochronology. The $2.6 \mathrm{Ma}$ and $0.78 \mathrm{Ma}$ NHG phase boundaries 
presented in our study represent the Matuyama/Gauss and Brunhes-Matuyama paleomagnetic boundaries, whereas the NHG Phase III boundary is based on paleomagnetic excursion events Top Olduvai (1.77 Ma) and Base Jaramillo (1.0 Ma) and biostratigraphy. These paloemagnetic boundaries and excursions have been identified in a number of deep sea cores and shallow drillings in the studied region (Fig. S1; Table S2).

It should be noted that in the northern Nansen Basin, Arctic Ocean, the chronostratigraphy is based on downlap of sequence boundaries onto oceanic basement of known age (Nikishin et al., 2018). As no Base Late Pleistocene boundary has been identified in this region the Miocene sequence boundary has been used when generating the Quaternary isopach map (Fig. 1, Fig. S2). Thereby, the Quaternary sediment thicknesses should be considered a maximum value. However, likewise to the other margin segments investigated in this study, we assume that the Miocene sediment input to the Arctic Ocean margin is very low compared to the sediment volumes deposited during the last 2.6 million years. In the North Sea TMF the Base Late Pleistocene boundary is also rather poorly constrained. We consider Stoker et al. (2005) to have the most reliable mapping of the Base Late Pleistocene sequence boundary in this region.

Published ice-rafted debris (IRD) records from the Yermak Plateau, the Fram Strait, the Mid-Norwegian and the British-Irish margins have been compiled (Fig. S3). The IRD records presented are all located nearby the paleo-EurIS marine margin, and is thus suggested to reflect size-variations in the KBIS (ODP sites 911, 908/909, 987), FIS (ODP sites 643, 644) and BIIS (IODP Site U1317E). It should be noted that the records are shown in weight percent, number of IRD grains etc. In addition, different grain sizes $(>2 \mathrm{~mm},>0.5 \mathrm{~mm},>0.125 \mathrm{~mm})$, has also been used.

Lastly, we have compiled glacial landform evidences of the existence of the EurIS (Table S3). Such evidences are Mega-Scale Glacial Lineations (MSGLs), formed beneath ice streams, 
and Glacigenic Debris Flows (GDFs), which are related to shelf-edge glaciations and ice streaming.

\section{SEDIMENT VOLUME AND SEDIMENTATION RATE ESTIMATES - METHODS}

\section{Quaternary sediment volumes and sedimentation rates}

In order to estimate sediment volumes the "extract by mask" and "surface volume" tools in the ArcGIS software (version 10.6) have been used. For this, the mid-oceanic spreading centers (Fig. 1) define the 0-m-thickness contour. Quaternary sediment volumes were first estimated for each margin segment (Table S4a) and then these sediment volumes were summed to represent erosion products derived from the KBIS, FIS and BIIS ice sheets (Table S4b). An average Quaternary sediment (dry) density of $1.80 \mathrm{~g} / \mathrm{cm}^{3}$, as estimated by Hjelstuen et al. (1996) and Fielder and Faleide (1996), has been used for converting sediment volumes into weight in tons.

For each main depocentre along the EurIS marine margin average Quaternary sedimentation rates (Table S5) have been estimated by using the above-described ArcGIS procedure, delineating the depositional area by the $200 \mathrm{~m}$ thickness contour (except for the Donegal TMF) and by utilizing the equation: (sediment volume / depositional area) : year. Maximum rates (Table S5) are also estimated by reading off maximum thickness in each depocentre from the ArcGIS-extracted isopach map.

Due to sparse age control in the northern part of the Arctic Ocean the Quaternary sediment volume should be considered an absolute maximum in this region. Elsewhere along the paleo-EurIS marine margin the sediment volume is anticipated rather well constrained. We have defined a 0 -m contour along the shelf edge and along the mid-oceanic ridge. This is also considered reliable, as e.g. seismic profiles across TMF systems along the western Svalbard-Barents Sea margins (Fig. S2) clearly show that they are terminating toward the oceanic spreading center.

Preglacial (55-2.7 Ma) sedimentation rates 
The pre-glacial sedimentation rates are, in regions where isopach maps exits (e.g. the western Barents Sea), based on estimated sediment volumes and depositional areas presented in published papers (Table S6). Elsewhere, such estimates are based on published seismic profiles and/or isopach maps, using sediment thickness information from the shelf edge regions. In Table S6 the method used to estimate sedimentation rates ("average" - based on sediment volumes and depositional areas and/or "linear" - based on seismic profiles and/or isopach maps) are indicated.

\section{Sedimentation rates and sediment discharge - the three glacial phases}

Commonly, in regions where isopach maps have been published for the three NHG phases defined in this study (e.g. Dowdeswell et al., 2010; Anell et al., 2012) information on sediment volumes, sediment thicknesses and depositional area have been estimated and corresponding average sedimentation rates and sediment discharge values have been calculated. In the present paper we use this information directly or if a new chronostratigraphy (Table S1) have been utilized, as for e.g. the western Barents Sea margin, average sediment rates and sediment discharge has been recalculated (Tables S6, S7). In areas where the above-mentioned information does not exist, shelf edge sediment thicknesses in published seismic profiles have been used.

(1)

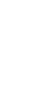

(1)

3


Table S1. Previously published information used in this study. Column 1 (from left): Number refer to number as annotated in Fig. S1 which show study area of the published papers listed in this table. Column 2: Study area/region in actual paper. Column 3: Figures and/or information from tables which have been used in present study. Column 4: Chronostratigraphic framework which has been used in present study. Column 5: Sediment velocities used in order to depth convert (to depth in meter) seismic profiles and/or isopach maps. Column 5: Information in the actual paper has been used: (i) to generate the Quaternary isopach map (QM), (ii) to estimate Quaternary sedimentation rates (GR), (iii) to estimate preQuaternary Cenozoic sedimentation rates (PR) or (iv) as background and/or supporting information (BI). Column 6: Authors of the published papers listed in this table.

Table S1-A. East Arctic Ocean margin

\begin{tabular}{|c|c|c|c|c|c|c|}
\hline $\begin{array}{c}\text { Id } \\
\text { (Fig. S1) }\end{array}$ & Region & Figure - Table & Chronology (based on) & Sediment velocities & Used for & Reference \\
\hline 1 & Nansen Basin & Fig. 5,6 & $\begin{array}{l}\text { Used chronology as presented in paper } \\
\text { (Downlap of sequence boundaries on } \\
\text { oceanic basement) }\end{array}$ & Velocities as given in paper & $\mathrm{QM}, \mathrm{GR}, \mathrm{PR}$ & Nikishin et al (2018) \\
\hline 2 & Nansen Basin & Fig. 2 & $\begin{array}{l}\text { Used chronology as presented in paper } \\
\text { (Regional seismic stratigraphy, ODP sites) }\end{array}$ & $\mathrm{N} / \mathrm{A}$ & QM & $\begin{array}{l}\text { Jokat and Micksch } \\
\text { (2004) }\end{array}$ \\
\hline 3 & Nansen Basin & Fig. 8 & $\begin{array}{l}\text { Used chronology as presented in paper } \\
\text { (Fig. 13) }\end{array}$ & Velocities as given in paper & QM & Engen et al. (2009) \\
\hline 4 & Yermak Plateau & Fig. 17a & $\begin{array}{l}\text { Used chronology as presented in paper } \\
\text { (Regional seismic stratigraphy, ODP sites) }\end{array}$ & Velocities as given in paper & QM & Geissler et al. (2011) \\
\hline 5 & Yermak Plateau & Fig. 3-6, 8-11 & $\begin{array}{l}\text { Used chronology as presented in paper } \\
\text { (Regional seismic stratigraphy, ODP sites) }\end{array}$ & Velocities as given in paper & QM & Geissler and Jokat (2004) \\
\hline 6 & Yermak Plateau & $\mathrm{N} / \mathrm{A}$ & $\begin{array}{l}\text { Used chronology as presented in paper } \\
\text { (Regional seismic stratigraphy, ODP sites) }\end{array}$ & $\mathrm{N} / \mathrm{A}$ & $\mathrm{BI}$ & Fransner et al. (2018) \\
\hline 7 & $\begin{array}{l}\text { N Svalbard } \\
\text { Margin }\end{array}$ & Table 1, 2 & $\begin{array}{l}\text { Used chronology as presented in paper } \\
\text { (Regional seismic stratigraphy, ODP sites) }\end{array}$ & $\mathrm{N} / \mathrm{A}$ & GR & Lasabuda et al. (2018) \\
\hline 8 & Fram Strait & Fig. 3-5 & $\begin{array}{l}\text { Used chronology as presented in paper } \\
\text { (Regional seismic stratigraphy, ODP sites) }\end{array}$ & $1800 \mathrm{~m} / \mathrm{s}$ (Geissler et al. 2011) & QM & Gebhardt et al. (2014) \\
\hline
\end{tabular}


Table S1-B. Western Svalbard margin

\begin{tabular}{|c|c|c|c|c|c|c|}
\hline $\begin{array}{c}\text { Id } \\
\text { (Fig. S1) }\end{array}$ & Region & Figure - Table & Chronology (based on) & Sediment velocities & Used for & Reference \\
\hline 9 & $\begin{array}{l}\text { Sjubrebanken } \\
\text { TMF }\end{array}$ & Fig. 11 & $\begin{array}{l}\text { Used chronology presented in paper } \\
\text { (Regional seismic stratigraphy, ODP sites } \\
911 \text { and 986) }\end{array}$ & 1800 m/s (Geissler et al. 2011) & QM & Sarkar et al. (2011) \\
\hline 10 & $\begin{array}{l}\text { Sjubrebanken } \\
\text { TMF }\end{array}$ & Fig. 6 & $\begin{array}{l}\text { Used chronology presented in paper (ODP } \\
\text { sites 910-912) }\end{array}$ & 1800 m/s (Geissler et al. 2011) & GR, PR & Mattingsdal et al. (2014) \\
\hline 9 & $\begin{array}{l}\text { Kongsfjorden } \\
\text { TMF }\end{array}$ & Fig. 11 & $\begin{array}{l}\text { Used chronology presented in paper } \\
\text { (Regional seismic stratigraphy, ODP sites } \\
911 \text { and } 986 \text { ) }\end{array}$ & 1800 m/s (Geissler et al. 2011) & QM & Sarkar et al. (2011) \\
\hline 10 & $\begin{array}{l}\text { Kongsfjorden } \\
\text { TMF }\end{array}$ & Fig. 6 & $\begin{array}{l}\text { Used chronology presented in paper (ODP } \\
\text { sites } 910-912 \text { ) }\end{array}$ & $1800 \mathrm{~m} / \mathrm{s}$ (Geissler et al. 2011) & GR, PR & Mattingsdal et al. (2014) \\
\hline 11 & Isfjorden TMF & Fig. 14 & $\begin{array}{l}\text { Use revised chronology by Laberg et al. } \\
(2010 ; 2012)\end{array}$ & $\begin{array}{l}2000 \mathrm{~m} / \mathrm{s} \text { (Solheim et al., } \\
1998)\end{array}$ & QM & Faleide et al. (1996) \\
\hline 12 & Isfjorden TMF & Fig. 3 & $\begin{array}{l}\text { Use revised chronology by Laberg et al. } \\
\text { (2010; 2012) (Knies et al. (2009), } \\
\text { paleomagnetism, amino acid) }\end{array}$ & $\begin{array}{l}2000 \mathrm{~m} / \mathrm{s} \text { (Solheim et al., } \\
1998)\end{array}$ & $\mathrm{BI}$ & Solheim et al. (1996) \\
\hline 13 & Isfjorden TMF & Fig. 3a & $\begin{array}{l}\text { Use revised chronology by Laberg et al. } \\
\text { (2010; 2012) (Knies et al. (2009), } \\
\text { paleomagnetism, amino acid) }\end{array}$ & $\begin{array}{l}2000 \mathrm{~m} / \mathrm{s} \text { (Solheim et al., } \\
1998)\end{array}$ & GR, PR & Amundsen et al. (2011) \\
\hline 11 & Bellsund TMF & Fig. 14 & $\begin{array}{l}\text { Use revised chronology by Laberg et al. } \\
\text { (2010; 2012) (Knies et al. (2009), } \\
\text { paleomagnetism, amino acid) }\end{array}$ & $\begin{array}{l}2000 \mathrm{~m} / \mathrm{s} \text { (Solheim et al., } \\
1998)\end{array}$ & QM & Faleide et al. (1996) \\
\hline 12 & Bellsund TMF & Fig. 3 & $\begin{array}{l}\text { Use revised chronology by Laberg et al. } \\
\text { (2010; 2012) (Knies et al. (2009), } \\
\text { paleomagnetism, amino acid) }\end{array}$ & $\begin{array}{l}2000 \mathrm{~m} / \mathrm{s} \text { (Solheim et al., } \\
1998)\end{array}$ & $\mathrm{BI}$ & Solheim et al. (1996) \\
\hline 13 & Bellsund TMF & Fig. 6a & $\begin{array}{l}\text { Use revised chronology by Laberg et al. } \\
\text { (2010; 2012) (Knies et al. (2009), } \\
\text { paleomagnetism, amino acid) }\end{array}$ & $\begin{array}{l}2000 \mathrm{~m} / \mathrm{s} \text { (Solheim et al., } \\
1998)\end{array}$ & GR, PR & Amundsen et al (2011) \\
\hline
\end{tabular}


Table S1-C. Western Barents Sea margin

\begin{tabular}{|c|c|c|c|c|c|c|}
\hline $\begin{array}{c}\text { Id } \\
\text { (Fig. S1) }\end{array}$ & Region & Figure - Table & Chronology & Sediment velocities & Used for & Reference \\
\hline 14 & Storfjorden TMF & Fig. 9, Table 2 & $\begin{array}{l}\text { Use revised chronology by Laberg et al. } \\
\text { (2010; 2012) (Knies et al. (2009), } \\
\text { paleomagnetism, amino acid) }\end{array}$ & $\begin{array}{l}\text { This study has already depth } \\
\text { converted maps }\end{array}$ & $\mathrm{QM}, \mathrm{GR}, \mathrm{PR}$ & Hjelstuen et al. (1996) \\
\hline 15 & $\begin{array}{l}\text { Bjørnøyrenna } \\
\text { TMF }\end{array}$ & Fig. 6, Table 2 & $\begin{array}{l}\text { Use revised chronology by Laberg et al. } \\
\text { (2010; 2012) (Knies et al. (2009), } \\
\text { paleomagnetism, amino acid) }\end{array}$ & $\begin{array}{l}\text { This study has already depth } \\
\text { converted maps }\end{array}$ & QM, GR, PR & $\begin{array}{l}\text { Fiedler and Faleide } \\
\text { (1996) }\end{array}$ \\
\hline
\end{tabular}

Table S1-D. Norwegian margin

\begin{tabular}{|c|c|c|c|c|c|c|}
\hline $\begin{array}{c}\text { Id } \\
\text { (Fig. S1) }\end{array}$ & Region & Figure - Table & Chronology & Sediment velocities & Used for & Reference \\
\hline 16 & $\begin{array}{l}\text { Mid Norwegian } \\
\text { Margin }\end{array}$ & $\begin{array}{l}\text { Fig. } 1 \text { and } \\
\text { Table } 1\end{array}$ & Used chronology presented in paper & Velocities as given in paper & QM, GR & Dowdeswell et al (2010) \\
\hline 17 & $\begin{array}{l}\text { North Sea TMF } \\
\text { (proximal) }\end{array}$ & $\begin{array}{l}\text { Table } 1 \text { and } \\
\text { Fig. } 4 \text { (dep } \\
\text { area) }\end{array}$ & Used chronology presented in paper & Velocities as given in paper & GR, QM & Hjelstuen et al. (2012) \\
\hline 18 & $\begin{array}{l}\text { North Sea TMF } \\
\text { (proximal) }\end{array}$ & Fig. 6c & Used chronology presented in paper & $2000 \mathrm{~m} / \mathrm{s}$ & QM & Stoker et al. (2005) \\
\hline 19 & $\begin{array}{l}\text { North Sea TMF } \\
\text { (proximal) }\end{array}$ & Fig. 4 & Used chronology presented in paper & $2000 \mathrm{~m} / \mathrm{s}$ & QM & Ottesen et al. (2012) \\
\hline 20 & Norway Basin & $\begin{array}{l}\text { Fig. } 4 \mathrm{~b} \text { and } \\
\text { new seismic } \\
\text { interpretation }\end{array}$ & Used chronology presented in paper & Velocities as given in paper & $\begin{array}{l}\text { GR (2.7-0.5 } \\
M a), P R, Q M\end{array}$ & $\begin{array}{l}\text { Hjelstuen and AnSeassen } \\
(2015)\end{array}$ \\
\hline 17 & Norway Basin & $\begin{array}{l}\text { Table 1, new } \\
\text { area estimates }\end{array}$ & Used chronology presented in paper & Velocities as given in paper & GR (0.5-0 Ma) & Hjelstuen et al. (2012) \\
\hline
\end{tabular}


Table S1-E. North Sea

\begin{tabular}{|c|c|c|c|c|c|c|}
\hline $\begin{array}{c}\text { Id } \\
\text { (Fig. S1) }\end{array}$ & Region & Figure - Table & Chronology & Sediment velocities & Used for & Reference \\
\hline 21 & North Sea & Fig. 4a, Table 1 & Used chronology presented in paper & $\begin{array}{l}2000 \mathrm{~m} / \mathrm{s} \text { ( } 1800 \mathrm{~m} / \mathrm{s} \text { used in } \\
\text { paper to estimate volume) }\end{array}$ & QM, GR & Ottesen et al. (2018) \\
\hline 22 & North Sea & Table 2 & Used chronology presented in paper & Velocities as given in paper & PR & Anell et al. (2012) \\
\hline 23 & North Sea & $\mathrm{N} / \mathrm{A}$ & Used chronology presented in paper & $\mathrm{N} / \mathrm{A}$ & $\mathrm{BI}$ & Sejrup et al. (1987) \\
\hline 24 & North Sea & $\mathrm{N} / \mathrm{A}$ & Used chronology presented in paper & $\mathrm{N} / \mathrm{A}$ & $\mathrm{BI}$ & Sejrup et al. (1995) \\
\hline 25 & North Sea & $\mathrm{N} / \mathrm{A}$ & Used chronology presented in paper & $\mathrm{N} / \mathrm{A}$ & $\mathrm{BI}$ & Sejrup et al. (2000) \\
\hline 26 & North Sea & Table 2, Fig. 2 & Used chronology presented in paper & Velocities as given in paper & GR & Reinardy et al. (2017) \\
\hline 27 & North Sea & $\mathrm{N} / \mathrm{A}$ & Used chronology presented in paper & $\mathrm{N} / \mathrm{A}$ & $\mathrm{BI}$ & Reinardy et al. (2018) \\
\hline
\end{tabular}

Table S1-F. British-Irish North Atlantic margin

\begin{tabular}{|c|l|c|c|c|c|c|}
\hline $\begin{array}{c}\text { Id } \\
\text { (Fig. S1) }\end{array}$ & \multicolumn{1}{|c|}{ Region } & $\begin{array}{c}\text { Figure - } \\
\text { Table }\end{array}$ & Chronology & Sediment velocities & Used for & Reference \\
\hline 18 & $\begin{array}{l}\text { British-Irish } \\
\text { margin }\end{array}$ & Fig. 6c \& 6b & Used chronology presented in paper & $2000 \mathrm{~m} / \mathrm{s}$ & QM, GR, PR & Stoker et al. (2005) \\
\hline
\end{tabular}


Table S2. Identified magnetostratigraphic events at selected sites and shallow borings along the EurIS marine margin. B/M: Brunhes-Matuyama; M/G: Matuyama-Gauss

\begin{tabular}{|c|c|c|}
\hline Site/Boring (Fig. S1) & Identified magnetostratigraphic events - Quaternary & Reference \\
\hline ODP site 908 & B/M boundary, Jaramillo Top, Jaramillo Base, Olduvai Top, Olduvai Base, M/G boundary & Myhre et al. (1995) \\
\hline ODP Site 909 & B/M boundary, Jaramillo Top, Jaramillo Base, M/G boundary & Myhre et al. (1995) \\
\hline ODP Site 910 & B/M boundary, Jaramillo Top, Jaramillo Base, Cobb Mountain Top & Myhre et al. (1995) \\
\hline ODP Site 911 & $\begin{array}{l}\text { B/M boundary, Jaramillo Top, Jaramillo Base, Cobb Mountain Top, Olduvai Top, Olduvai Base, M/G } \\
\text { boundary }\end{array}$ & Myhre et al. (1995) \\
\hline ODP Site 986 & B/M boundary, Jaramillo Top, Jaramillo Base, Olduvai Top, Olduvai Base, Reunion & Channell et al. (1999) \\
\hline ODP Site 644 & B/M boundary, Jaramillo Top, Jaramillo Base, Olduvai Top, Olduvai Base, M/G boundary & Eldholm et al. (1987) \\
\hline ODP Site 643 & B/M boundary, Jaramillo Top, Jaramillo Base, Olduvai Top, Olduvai Base, M/G boundary & Eldholm et al. (1987) \\
\hline ODP Site 642 & B/M boundary, Jaramillo Top, Jaramillo Base, Olduvai Top, Olduvai Base, M/G boundary & Eldholm et al. (1987) \\
\hline $77 / 2$ & B/M boundary, Jaramillo event & Stoker et al. (1983) \\
\hline $81 / 26$ & $\mathrm{~B} / \mathrm{M}$ boundary, Jaramillo event & $\begin{array}{l}\text { Stoker et al. (1983), Sejrup et } \\
\text { al. (1987) }\end{array}$ \\
\hline $89-03$ & B/M boundary, Jaramillo event, Cobb Mountain event & Sejrup et al. (1995) \\
\hline Southern North Sea & Olduvai Top, Olduvai Base, Reunion, M/G boundary & Kuhlmann et al. (2006) \\
\hline
\end{tabular}


Table S3. Glacial landform evidences of ice sheets and ice streams, EurIS marine margin. Increased font size indicates "more prominent" and/or “increased effect”. GDF: Glacigenic Debris Flows, ICE: Grounded ice erosion, P: Iceberg plough marks, M: Mega-scale glacial lineations.

\begin{tabular}{|c|c|c|c|c|c|c|c|c|}
\hline $\begin{array}{c}\text { Time period } \\
\text { (Ma) }\end{array}$ & $\begin{array}{c}\text { N Svalbard } \\
\text { Margin }^{1}\end{array}$ & $\begin{array}{l}\text { NW Svalbard } \\
\text { Margin }^{2,3}\end{array}$ & $\begin{array}{c}\text { W Svalbard } \\
\text { Margin }^{4}\end{array}$ & $\begin{array}{l}\text { NW Barents } \\
\text { Sea Margin }\end{array}$ & $\begin{array}{l}\text { SW Barents } \\
\text { Sea Margin }\end{array}$ & $\begin{array}{c}\text { Mid-Norwegian } \\
\text { margin }^{7}\end{array}$ & $\begin{array}{c}\text { North Sea } \\
\text { TMF }^{8,9}\end{array}$ & North Sea ${ }^{10,11,12}$ \\
\hline $0.0-0.78$ & GDF & ICE & GDF & GDF & GDF & $M, P$ & GDF & GDF, P, MSGL \\
\hline $0.78-1.0$ & GDF & ICE & GDF & GDF & GDF & $\mathrm{M}, \mathrm{P}$ & GDF & GDF, P, MSGL \\
\hline $1.0-1.5$ & GDF & ICE & GDF & GDF & GDF & $\mathrm{M}, \mathrm{P}$ & GDF & GDF, P, MSGL \\
\hline $1.5-2.0$ & GDF & GDF & GDF (from 1.8 Ma?) & GDF (from 1.8 Ma?) & & $\mathrm{M}, \mathrm{P}$ & & GDF, P,MSGL \\
\hline $2.0-2.6$ & GDF & GDF & & & & $\mathrm{M}, \mathrm{P}$ & & GDF, $P$ \\
\hline
\end{tabular}

${ }^{1}$ Lasabuda et al. (2018), ${ }^{2}$ Sakar et al. (2011), ${ }^{3}$ Mattingsdal et al. (2014), ${ }^{4}$ Solheim et al. (1998), ${ }^{5}$ Laberg et al. (2010), ${ }^{6}$ Waage et al. (2018), ${ }^{7}$ Montelli et al. (2017), ${ }^{8} \mathrm{Hjelstuen}$ and Grinde (2016), ${ }^{9}$ Nygård et al. (2005), ${ }^{10}$ Ottesen et al. (2018), ${ }^{11}$ Reinardy et al. (2017), ${ }^{12}$ Rea et al. (2018) 
Table S4 a. Estimated Quaternary sediment volumes for the different segments of the EurIS marine margin

\begin{tabular}{|c|c|c|c|c|}
\hline Margin segment & $\begin{array}{l}\text { Sediment volum } \\
\left(10^{3} \mathrm{~km}^{3}\right)\end{array}$ & $\begin{array}{c}\text { Depositional area } \\
\left(10^{3} \mathrm{~km}^{2}\right)\end{array}$ & $\begin{array}{c}\% \text { sediment volum } \\
\text { of total }\end{array}$ & $\begin{array}{c}\text { Weight } \\
\left(10^{14} \text { tons }\right)\end{array}$ \\
\hline East Arctic Ocean margin & 743 & 952 & 41 & 13,4 \\
\hline Western Svalbard-Barents Sea margin & 591 & 476 & 33 & 10,6 \\
\hline Norway margin & 265 & 619 & 15 & 4,8 \\
\hline Irish-British North Atlantic margin & 46,2 & 406 & 3 & 0,8 \\
\hline North Sea & 143 & 326 & 8 & 2,6 \\
\hline North Sea (UK sector) & 53,9 & 129 & & \\
\hline North Sea (Norwegian sector) & 89,1 & 197 & & \\
\hline Totalt & 1788,2 & 2779 & 100 & 32,2 \\
\hline
\end{tabular}

Table S4 b. Estimated Quaternary sediment volumes along the marine margins of the paleo-KBIS, paleo-FIS and paleo-BIIS ice sheets.

\begin{tabular}{lcccc}
\hline Ice sheet & $\begin{array}{c}\text { Sediment volum } \\
\left(10^{3} \mathrm{~km}^{3}\right)\end{array}$ & $\begin{array}{c}\text { Depositional area } \\
\left(10^{3} \mathrm{~km}^{2}\right)\end{array}$ & $\begin{array}{c}\text { \% sediment volum } \\
\text { of total }\end{array}$ & $\begin{array}{c}\text { Weight } \\
\left(10^{14} \text { tons }\right)\end{array}$ \\
\hline KBIS & 1334 & 1428 & 75 & 24,0 \\
FIS & 354,1 & 816 & 20 & 5,4 \\
BIIS & 100,1 & 535 & 5 & 1,8 \\
\hline Totalt & 1788,2 & 2779 & 100 & 32,2 \\
\hline
\end{tabular}


Table S5. Estimated Quaternary sediment volumes and sediment rates in the main depocentres along the EurIS marine margin. For location see Fig. 1.

\begin{tabular}{|c|c|c|c|c|c|c|c|}
\hline Region & $\begin{array}{c}\text { Depositional } \\
\text { area }\left(10^{3} \mathrm{~km}^{2}\right)\end{array}$ & $\begin{array}{l}\text { Sediment volume } \\
\left(10^{3} \mathrm{~km}^{3}\right)\end{array}$ & $\begin{array}{c}\text { Maximum } \\
\text { thickness (m) }\end{array}$ & $\begin{array}{c}\text { Average } \\
\text { thickness }(\mathrm{m})\end{array}$ & $\begin{array}{c}\text { Maximum rate } \\
\text { (cm/kyr) }\end{array}$ & $\begin{array}{c}\text { Average rate } \\
(\mathrm{cm} / \mathrm{kyr})\end{array}$ & $\begin{array}{c}\text { Delineated by } \\
\text { contour }(\mathrm{m})\end{array}$ \\
\hline North Nansen Basin & 278 & 299 & 2300 & 1080 & 88 & 41 & 200 \\
\hline South Nansen Basin & 128 & 80 & 1250 & 623 & 48 & 24 & 200 \\
\hline Fram Strait & 13 & 3 & 590 & 241 & 23 & 9 & 200 \\
\hline Svalbard Margin & 16 & 18 & 1680 & 1090 & 65 & 42 & 200 \\
\hline Storfjorden TMF & 56 & 102 & 4500 & 1780 & 173 & 69 & 200 \\
\hline Bjørnøуa TMF & 281 & 379 & 3600 & 1340 & 140 & 52 & 200 \\
\hline Mid Norwegian margin & 114 & 58 & 1500 & 517 & 58 & 20 & 200 \\
\hline North Sea TMF & 64 & 47 & 1700 & 734 & 65 & 28 & 200 \\
\hline Norway Basin & 155 & 68 & 1300 & 442 & 50 & 17 & 200 \\
\hline North Sea & 203 & 81 & 1057 & 400 & 41 & 15 & 200 \\
\hline British-Irish margin & 528 & 11 & 252 & 220 & 10 & 8 & 0 \\
\hline
\end{tabular}


Table S6. Estimated sedimentation rates along the EurIS marine margin.

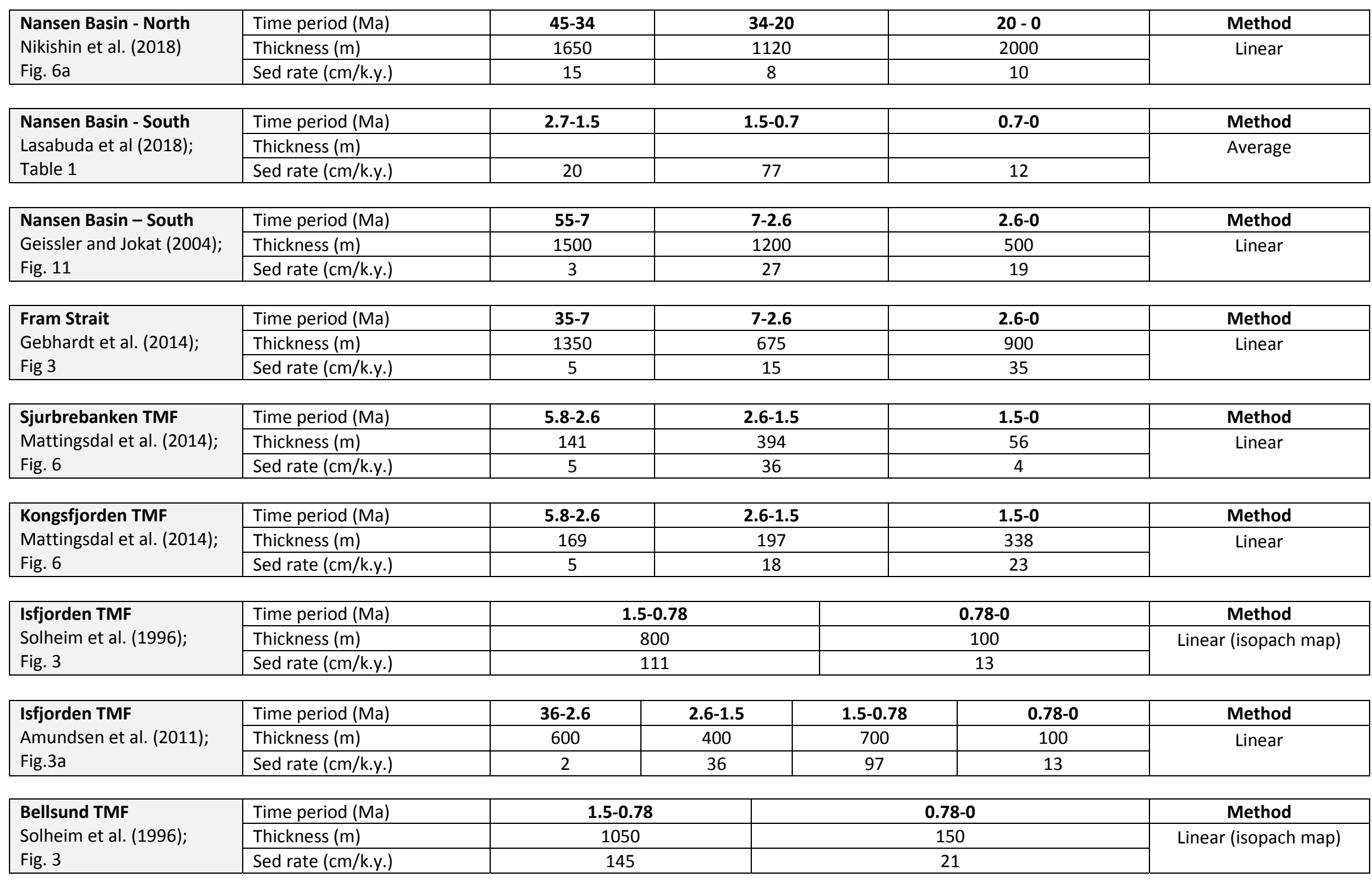




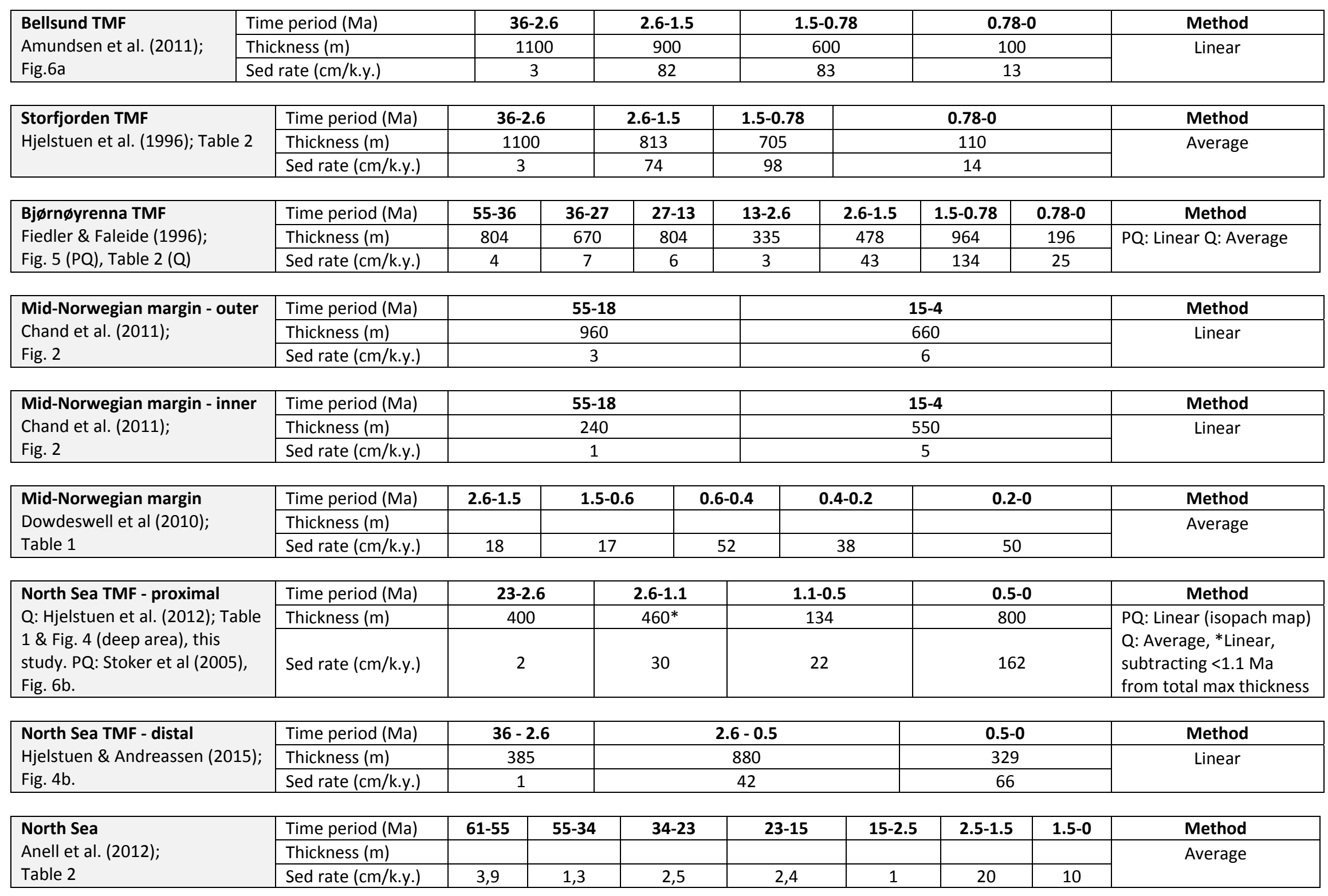




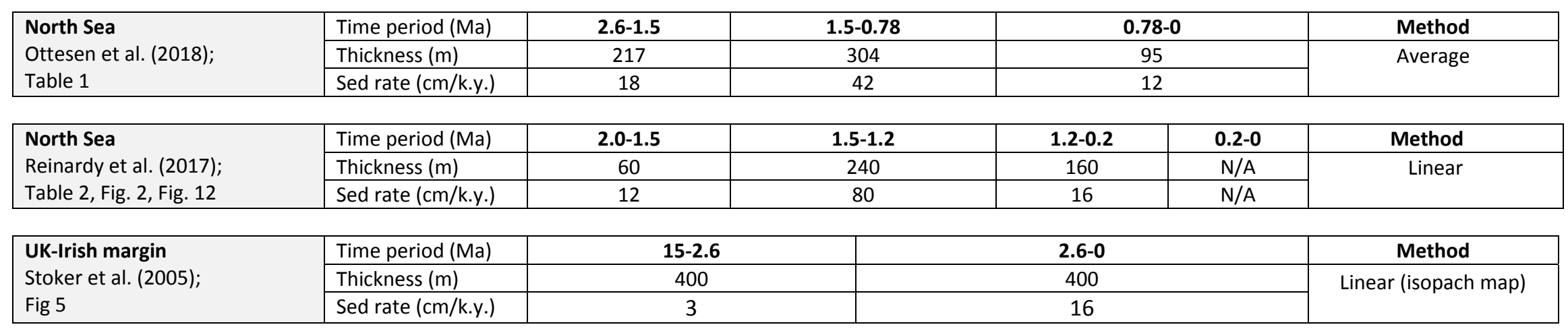


Table S7. Estimated sediment discharge $\left(10^{3} \mathrm{~m}^{3} / \mathrm{yr}\right)$.

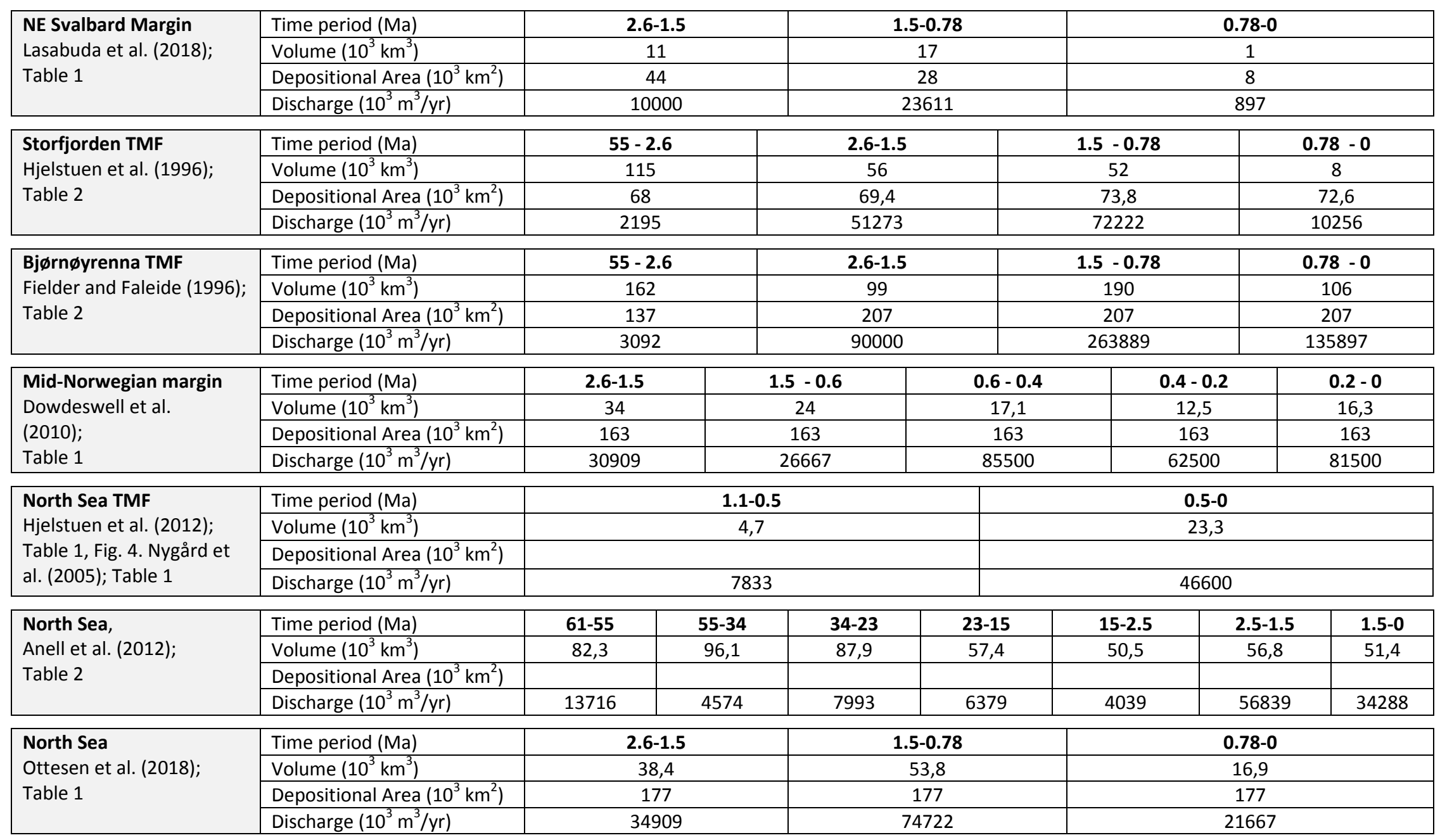




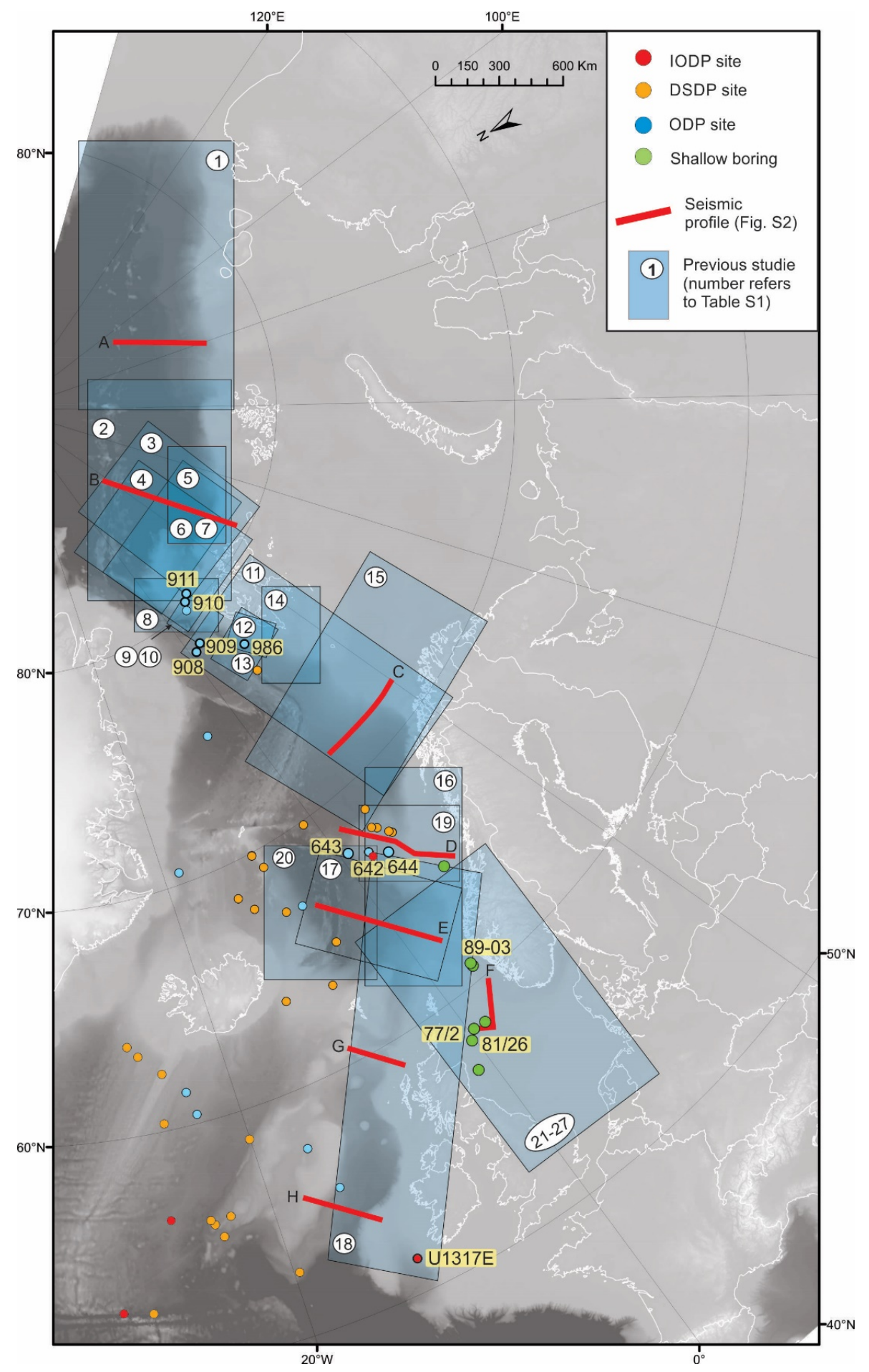

Figure S1. Overview of study areas in the published papers utilized in the present paper. Location of seismic profiles shown in Fig. S2, IODP-DSDP-ODP sites and shallow borings are shown. 

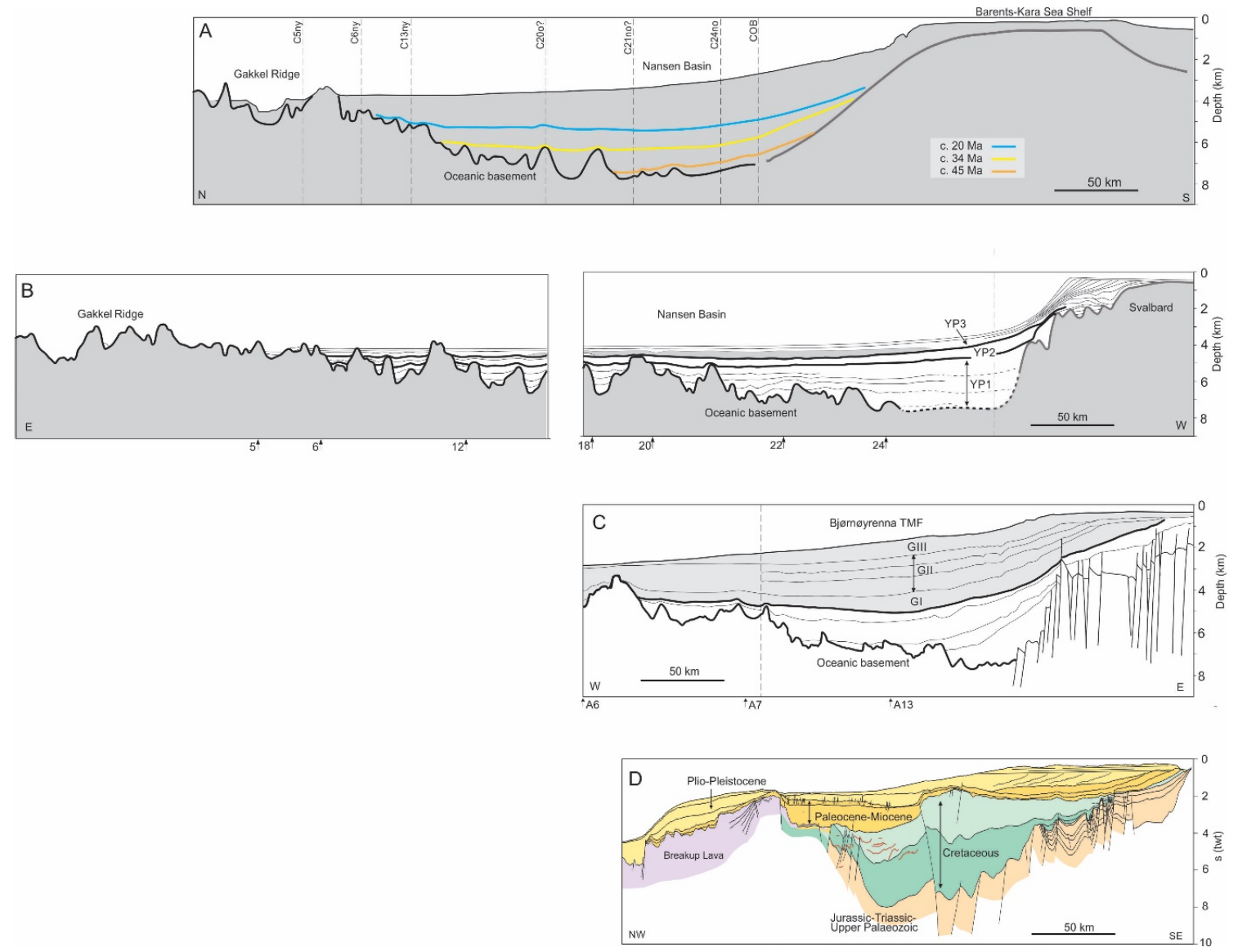

Figure S2. Line-drawing of seismic profiles showing typical geological settings along the paleo-EurIS marine margin. See Fig. S1 for profile location. (A) Northern Nansen Basin. COB: Continent-Ocean Boundary, C: Magnetic Cron (Modified from Nikishin et al. (2018)). (B) Southern Nansen Basin. YP1 (Eocene-Oligocene), YP2 (Miocene) YP3 (Pleistocene) are identified seismic units, A: Magnetic seafloor spreading anomalies (Modified from Jokat and Micksch (2004)), (C) Bjørnøyrenna TMF. GI (2.7-1.5 Ma), GII (1.5-0.7 Ma) and GIII (0.7-0 Ma) are identified seismic units. A: Magnetic seafloor spreading anomalies (Fiedler and Faleide, 1996). (D) Mid Norwegian margin (Modified from Blystad et al., 1995). 

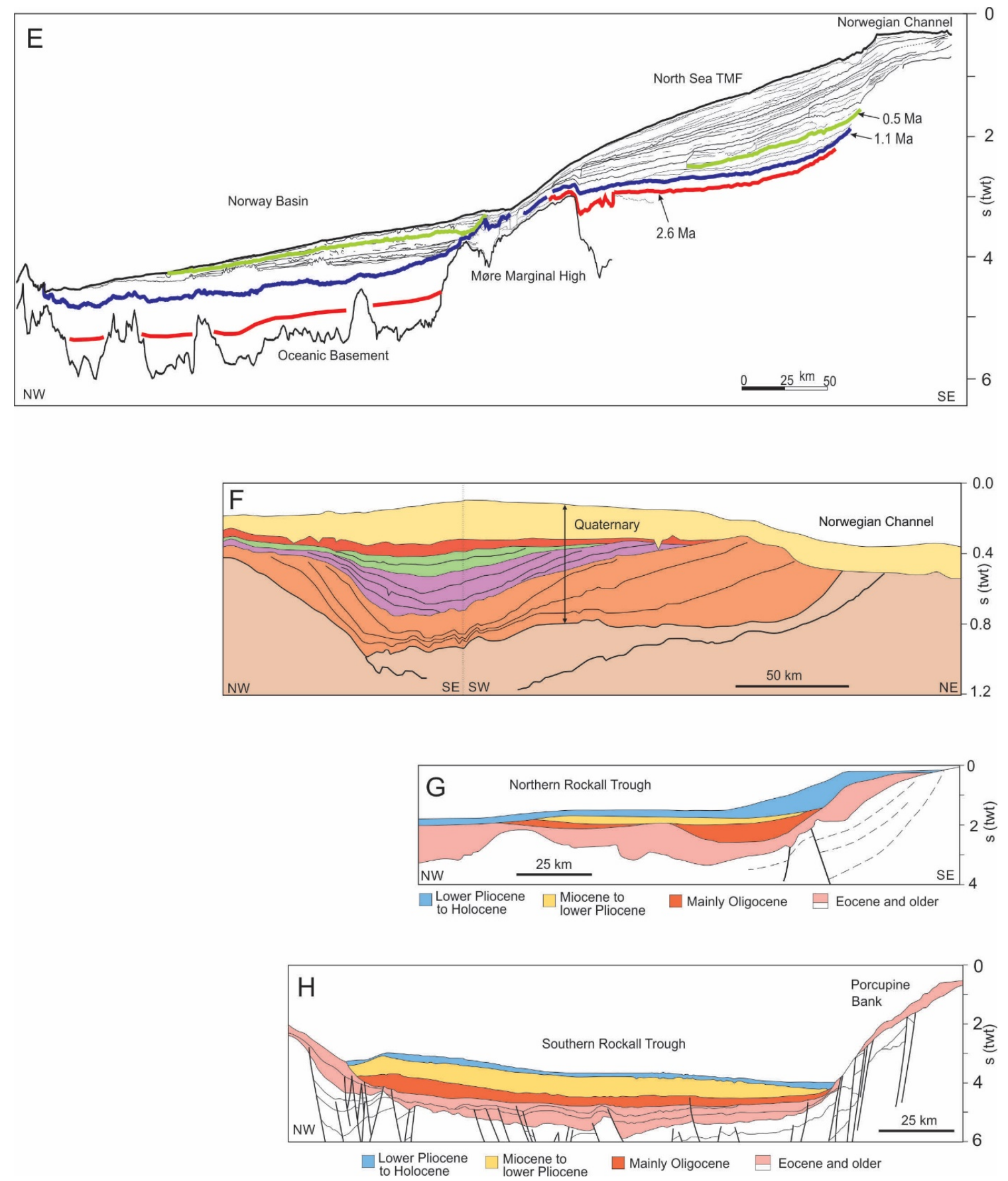

Figure S2 cont. (E) North Sea TMF and Norway Basin. (Modified from Hjelstuen et al. (2012)). (F) North Sea. (Modified from Baig (2018)), (G) British-Irish margin - north. (Modified from Stoker et al. (2005)), (H) British-Irish margin - south (Modified from Stoker et al., (2005)). 


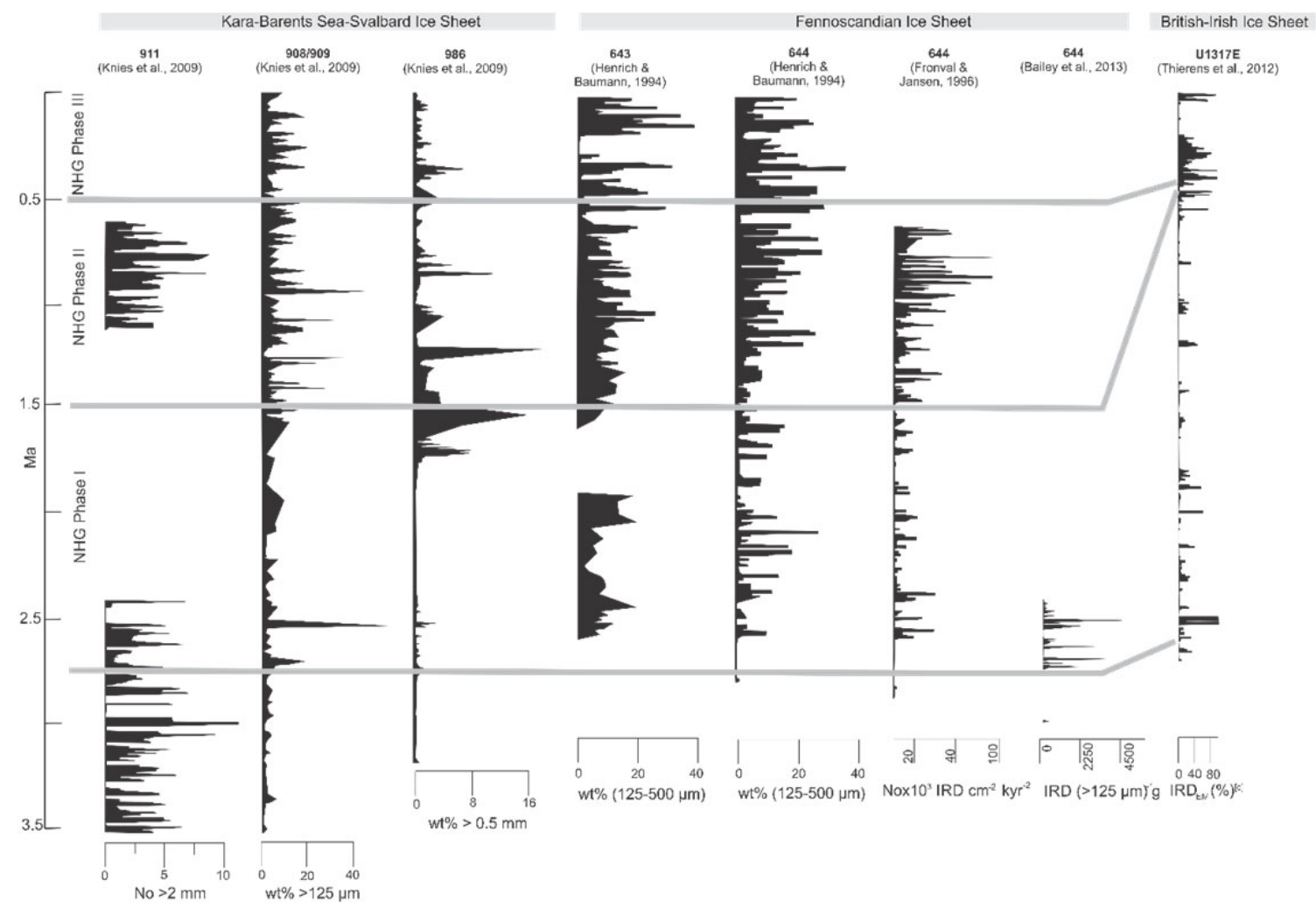

Figure S3. IRD records from the Svalbard, Mid-Norwegian and British-Irish margins. Location of ODP and IODP sites in Fig. S1. Compilation is based on Knies et al. (2009), Henrich and Baumann (1994), Fronval and Jansen (1996), Bailey et al. (2013) and Thierens et al. (2012). 


\section{REFERENCE CITED}

Amundsen, I.M.H., Blinova, M., Hjelstuen, B.O., Mjelde, R., and Haflidason, H., 2011, The Cenozoic western Svalbard margin - sediment geometry and sedimentary processes in an area of ultraslow oceanic spreading: Marine Geophysical Research, v. 32, p. 441-453, DOI 10.1007/s11001-011-9127-z.

Anell, I., Thybo, H., and Rasmussen, E., 2012, A synthesis of Cenozoic sedimentation in the North Sea: Basin Research, v. 24, p. 154-179, https://doi.org/10.1111/j.13652117.2011.00517.x.

Baig, I., 2018. Burial and thermal histories of sediments in the southwestern Barents Sea and North Sea areas: evidence from integrated compaction, thermal maturity and seismic stratigraphic analyses. PhD thesis, University of Oslo, Department of Geosciences, $165 \mathrm{pp}$.

Bailey, I., Hole, G.M., Foster, G.L., Wilson, P.A., Storey, C.D., Trueman, C.N., and Raymo, M.E., 2013, An alternative suggestion for the Pliocene onset of major northern hemisphere glaciation based on the geochemical provenance of North Atlantic Ocean ice-rafted debris: $\begin{array}{llllll}\text { Quaternary } & \text { Science } & \text { Reviews, } & \text { v. } & 75, & \text { p. }\end{array}$ https://doi.org/10.1016/j.quascirev.2013.06.004.

Blystad, P., Brekke, H., Færseth, R.B., Larsen, B.T., Skogseid, J., and Tørudbakken, B., 1995, Structural elements of the Norwegian continental shelf. Part II: The Norwegian Sea region: Norwegian Petroleum Directorate Bulletin, v. 8, 45 pp.

Chand, S., Rise, L., Knies, J., Haflidason, H., Hjelstuen, B.O., and Bøe, L., 2011, Stratigraphic development of the south Vøring Margin (Mid-Norway) since early Cenozoic time and its influence on subsurface fluid flow: Marine and Petroleum Geology, v. 28, p. 1350-1363, https://doi.org/10.1016/j.marpetgeo.2011.01.005. 
Channell, J.E.T., Smelror, M., Jansen, E., Higgins, S., Lehman, B., Eidvin, T., Solheim, A., 1999, Age models for glacial fan deposits off East Greenland and Svalbard (ODP Site 986 and Site 987). In: Raymo, M., Jansen, E., Blum, P., Herbert, T.D. (Eds.), Proceeding Ocean Drilling Program. Scientific Results, vol. 162, pp. 149-166. Ocean Drilling Program, College Station, TX. doi:10.2973/odp.proc.sr.162.008.1999

Dowdeswell, J.A., Ottesen, D., and Rise, L., 2010. Rates of sediment delivery from the Fennoscandian Ice Sheet through an ice age: Geology, v. 38, p. 3-6. doi: https://doi.org/10.1130/G25523.1

Eldholm, O., Thiede, J., Taylor, E., et al., 1987, Proceedings of the Ocean Drilling Program, Initial Reports, Leg 104, 1141 pp, Ocean Drilling Program, College Station, Texas, USA. doi:10.2973/odp.proc.ir.104.1987

Engen, Ø., Gjengedal, J.A., Faleide, J.I., Kristoffersen, Y., and Eldholm, O., 2009, Seismic stratigraphy and sediment thickness of the Nansen Basin, Arctic Ocean: Geophysical Journal International, v. 176, p. 805-821, https://doi.org/10.1111/j.1365246X.2008.04028.x.

Faleide, J.I., Solheim, A., Fiedler, A., Hjelstuen, B.O., Andersen, E.S., and Vanneste, K., 1996, Late Cenozoic evolution of the western Barents Sea-Svalbard continental margin: Global and Planetary Change, v. 12, p. 53-74, https://doi.org/10.1016/09218181(95)00012-7.

Fiedler, A., and Faleide, J.I., 1996, Cenozoic sedimentation along the southwestern Barents Sea margin in relation to erosion and uplift of the shelf: v. 12, p. 75-93, https://doi.org/10.1016/0921-8181(95)00013-5.

Fransner, O., Noormets, R., Flink, A.E., Hogan, K.A., Dowdeswell, J.A., 2018, Sedimentary processes on the continental slope off Kvitøya and Albertini troughs north of Nordaustlandet, Svalbard - The importance of structural-geological setting in trough- 
mouth fan development: Marine Geology, v. 402, p. 194-208, https://doi.org/10.1016/j.margeo.2017.10.008.

Fronval, T., and Jansen, E., 1996, Late Neogene paleoclimates and paleoceanography in the Iceland-Norwegian Sea: Evidence from the Iceland and Vøring Plateau. In: Thiede, J., et al., Proceedings of the Ocean Drilling Program, Scientific Results, v. 151, p. 455-468.

Gebhardt, A.C., Geissler, W.H., Matthiessen, J., and Jokat, W., 2014, Changes in current patterns in the Fram Strait at the Pliocene/Pleistocene boundary: Quaternary Science Reviews, v. 92, p. 179-189, https://doi.org/10.1016/j.quascirev.2013.07.015.

Geissler, W. H., Jokat, W., and Brekke, H., 2011. The Yermak Plateau in the Arctic Ocean in the light of reflection seismic data-implication for its tectonic and sedimentary evolution: Geophysical Journal International, v. 187, p. 1334-1362, https://doi.org/10.1111/j.1365246X.2011.05197.x.

Geissler, W.H., and Jokat, W., 2004, A geophysical study of the northern Svalbard continental margin. Geophysical Journal International, v. 158, p. 50-66, https://doi.org/10.1111/j.1365-246X.2004.02315.x.

Henrich, R., and Baumann, K.-H., 1994, Evolution of the Norwegian Current and the Scandinavian Ice Sheets during the past 2.6 m.y.: evidence from ODP Leg 104 biogenic carbonat and terrigenous records: Palaeogeography, Palaeoclimatology, Palaeoecology, v. 108, p. 75-94, https://doi.org/10.1016/0031-0182(94)90023-X.

Hjelstuen, B.O., and Andreassen, E.V, 2015, North Atlantic deep water processes and depositional environments: A study of the Cenozoic Norway Basin: Marine and Petroleum Geology, v. 59, p. 429-441, https://doi.org/10.1016/j.marpetgeo.2014.09.011.

Hjelstuen, B.O., Elverhøi, A., and Faleide, J.I., 1996, Cenozoic erosion and sediment yield in the drainage area of the Storfjorden Fan: Global and Planetary Change, v. 12, p. 95-117, https://doi.org/10.1016/0921-8181(95)00014-3. 
Hjelstuen, B.O., and Grinde, S., 2016, 3D seismic investigation of Pleistocene Mass Transport Deposits and Glacigenic Debris Flows on the North Sea Fan, NE Atlantic Margin. In: Lamarche, $G$ et al. (eds) Submarine Mass Movements and Their Consequences, Advances in Natural and Technological Hazard Research, pp 265-272.

Hjelstuen, B.O., Nygård, A., Sejrup, H.P., and Haflidason, H., 2012, Quaternary denudation of southern Fennoscandia - evidence from the marine realm: Boreas, v. 41, p. 379-390, https://doi.org/10.1111/j.1502-3885.2011.00239.x.

Jokat, W., and Micksch, U., 2004. Sedimentary structure of the Nansen and Amundsen basins, Arctic Ocean: Geophysical Research Letter, v. 31, L02603, doi: 10.1029/2003GL018352.

Knies, J., Matthiessen, J., Vogt, C., Laberg, J.S., Hjelstuen, B.O., Smelror, M., Larsen, E., Andreassen, K., Eidvin, T., and Vorren, T., 2009, The Plio-Pleistocene glaciations of the Barents Sea-Svalbard region: a new model based on revised chronostratigraphy: $\begin{array}{lllll}\text { Quaternary } & \text { Science } & \text { Reviews, } & \text { v. } & \text { 28, }\end{array}$ https://doi.org/10.1016/j.quascirev.2008.12.002.

Kuhlmann, G., Langereis, C., Munsterman, D., van Leeuwen, R.J., Verreussel, R., Meulenkamp, J., Wong, T., 2006, Chronostratigraphy of Late Neogene sediments in the southern North Sea Basin and paleoenvironmental interpretations: Palaeogeography, Palaeoclimatology, Palaeoecology, $\quad$ v. $\quad 239, \quad$ p. $\quad 426-455$, https://doi.org/10.1016/j.palaeo.2006.02.004.

Laberg, J.S., Andreassen, K., Knies, J., Vorren, T.O., and Winsborrow, M., 2010, Late Pliocene-Pleistocene development of the Barents Sea Ice Sheet: Geology, v. 38, p. 107110, https://doi.org/10.1130/G30193.1. 
Laberg, J.S., Andreassen, K., and Vorren, T.O., 2012, Late Cenozoic erosion of the highlatitude southwestern Barents Sea shelf revisited: Geological Society American Bulletin, v. 124, p. 77-99, https://doi.org/10.1130/B30340.1.

Lasabuda, A., Geissler, W. H., Laberg, J. S., Knutsen, S. M., Rydningen, T.A., and Berglar, K., 2018, Late Cenozoic erosion estimates for the northern Barents Sea: Quantifying glacial sediment input to the Arctic Ocean: Geochemistry, Geophysics, Geosystems, v. 19, p. 4876-4903, https://doi.org/10.1029/2018GC007882.

Mattingsdal, R., Knies, J., Andreassen, K., Fabian, K., Husum, K., Grøsfjeld, K., and De Schepper, S., 2014, A new $6 \mathrm{Myr}$ stratigraphic framework for the Atlantic-Arctic Gateway: Quaternary Science Reviews, v. 92, p. 170-178, https://doi.org/10.1016/j.quascirev.2013.08.022.

Montelli, M., Dowdeswell, J.A., Ottesen, D., and Johansen, S.E., 2017, Ice-sheet dynamics through the Quaternary on the mid-Norwegian continental margin inferred from 3D seismic data: Marine and Petroleum Geology, v. 80, p. 228-242, https://doi.org/10.1016/j.marpetgeo.2016.12.002.

Myhre, A., Thiede, J., Firth, J.A. (Eds.), 1995, Proceedings of the Ocean Drilling Program. Initial Reports, Leg 151, 951 pp. Ocean Drilling Program, College Station, Texas, USA, doi:10.2973/odp.proc.ir.151.1995.

Nikishin, A.M., Gaina, C., Petrov, E.I., Malyshev, N.A., and Freiman. S.I., 2018, Eurasia Basin and Gakkel Ridge, Arctic Ocean: Crustal asymmetry, ultra-slow spreading and continental rifting revealed by new seismic data: Tectonophysics, v. 746, p. 64-82, https://doi.org/10.1016/j.tecto.2017.09.006.

Nygård, A., Sejrup, H.P., Haflidason, H., and Bryn, P., 2005, The glacial North Sea Fan, southern Norwegian Margin: architecture and evolution from the upper continental slope to 
the deep-sea basin: Marine and Petroleum Geology, v. 22, p. 71-84, https://doi.org/10.1016/j.marpetgeo.2004.12.001.

Ottesen, D., Batchelor, C.L., Dowdeswell, J.A., and Løseth, H., 2018, Morphology and pattern of Quaternary sedimentation in the North Sea Basin $\left(52-62^{\circ} \mathrm{N}\right)$ : Marine and Petroleum Geology, v. 98, p. 836-859, https://doi.org/10.1016/j.marpetgeo.2018.08.022.

Ottesen, D., Dowdeswell, J.A., Rise, L., and Bugge, T., 2012, Large-scale development of the mid-Norwegian shelf over the last three million years and potential for hydrocarbon reservoirs in glacial sediments: Geological Society, London, Special Publications, v. 368, p. 53-73, https://doi.org/10.1144/SP368.6.

Rea, B.R., Newton, A.M.W., Lamb, R.M., Harding, R., Bigg, G.R., Rose, P., Spagnolo, M., Huuse, M., Cater, John M.L., Archer, S., Buckley, F., Halliyeva, M., Huuse, J., Cornwell, D.G., Brocklehurst, S.H., Howell, J.A., Extensive marine-terminating ice sheets in Europe from 2.5 million years ago, 2018, v. $4 ., 11$ pp., 10.1126/sciadv.aar8327

Reinardy, B.T.I., Hjelstuen, B.O., Sejrup, H.P., Augedal, H., and Jørstad, A., 2017, Late Pliocene-Pleistocene environments and glacial history of the northern North Sea: $\begin{array}{lllll}\text { Quaternary } & \text { Science } & \text { Reviews, } & \text { v. } & 158,\end{array}$ https://doi.org/10.1016/j.quascirev.2016.12.022.

Reinardy, B.T.I., Sejrup, H.P., Hjelstuen, B.O., King, E., and Augedal, H., 2018, A Quaternary aminostratigraphy constraining chronology of depositional environments in the North Sea Basin: Marine Geology, v. 402, p. 139-152, https://doi.org/10.1016/j.margeo.2017.11.004.

Rise, L., Ottesen, D., Longva, O., Solheim, A., Andersen, E.S., and Ayers, S., 2006, The Sklinnadjupet slide and its relation to the Elsterian glaciation on the mid-Norwegian margin: Marine and Petroleum Geology, v. 23, p. 569-583, https://doi.org/10.1016/j.marpetgeo.2006.05.005. 
Sarkar, S., C., Chabert, A., Masson, D.G., Minshull, T.A., and Westbrook, and G.K., 2011, Switching of a paleo-ice stream in northwest Svalbard: Quaternary Science Reviews, v. 30, p. 1710-1725, https://doi.org/10.1016/j.quascirev.2011.03.013.

Sejrup, H. P., E. Larsen, J. Landvik, E. L. King, H. Haflidason, and A. Nesje, 2000, Quaternary glaciations in southern Fennoscandia: evidence from southwestern Norway and the northern North Sea region: Quaternary Science Reviews, v. 19, p. 667-685, https://doi.org/10.1016/S0277-3791(99)00016-5.

Sejrup, H. P., I. Aarseth, H. Haflidason, R. Løvlie, Å. Bratten, G. Tjøstheim, C. F. Forsberg, and K. I. Ellingsen, 1995, Quaternary of the Norwegian Channel: glaciation history and palaeoceanography: Norsk Geologisk Tidsskrift, v. 75, p. 65-87.

Sejrup, H. P., I. Aarseth, K. L. Ellingsen, E. Reither, E. Jansen, R. Løvlie, A. Bent, J. Brigham-Grette, E. Larsen, and M. Stoker, 1987, Quaternary stratigraphy of the Fladen area, central North Sea: A multidisciplinary study: Journal of Quaternary Science, v. 2, p. 35-58, https://doi.org/10.1002/jqs.3390020105.

Solheim, A., Andersen, E.S., Elverhøi, A., and Fiedler, A., 1996, Late Cenozoic depositional history of the western Svalbard continental shelf, controlled by subsidence and climate, Global and Planetary Change, v. 12, 135-148, https://doi.org/10.1016/09218181(95)00016-X.

Solheim, A., Faleide, J.I., Andersen, E.S., Elverhøi, A., Forsberg, C.F., Vanneste, K., Uenzelmann-Neben, G., and Channell, J.E.T., 1998, Late Cenozoic seismic stratigraphy and glacial geological development of the Svalbard-Barents Sea continental margins. Quaternary Science Review, v. 17, p. 155-184, https://doi.org/10.1016/S02773791(97)00068-1.

Stoker, M.S., Praeg, D., Hjelstuen, B.O., Laberg, J.S., Nielsen, T., and Shannon, P.M., 2005, Neogene stratigraphy and the sedimentary and oceanographic development of the NW 
European Atlantic margin: Marine and Petroleum Geology, v. 22, p 977-1005, https://doi.org/10.1016/j.marpetgeo.2004.11.007.

Stoker, M.S., Skinner, A.C., Fyfe, J.A., Long, D., 1983, Paleomagnetic evidence for early Pleistocene in the central and northern North Sea. Nature, v. 304, p. 332-334.

Thierens, M., Pirlet, H., Colin, C., Latruwe, K., Vanhaecke, F., Lee, J.R., Stuut, J.-B., Titschack, J., Huvenne, V.A.I., Dorschel, B., Wheeler, A.J., and Henriet, J.-P., 2012, Icerafting from the British-Irish ice sheet since the earliest Pleistocene (2.6 million years ago): implications for long-term mid-latitudinal ice-sheet growth in the North Atlantic region: Quaternary Science Reviews, v. 44, p. 229-240, https://doi.org/10.1016/j.quascirev.2010.12.020.

Waage, M., Bünz, S., Bøe, R., and Mienert, J., 2018, High-resolution 3D seismic exhibits new insights into the middle-late Pleistocene stratigraphic evolution and sedimentary processes of the Bear Island trough mouth fan: Marine Geology, v. 403, p. 139-149, https://doi.org/10.1016/j.margeo.2018.05.006. 